\title{
Analysis of the Acceleration Process of SEPs by an Interplanetary Shock for Bastille Day Event
}

\author{
G.M.Le ${ }^{1,2} \dagger$, and Y.B.Han ${ }^{1}$ \\ ${ }^{1}$ The National Astronomical Observatories, Chinese Academy of Sciences, Beijing 100012 \\ ${ }^{2}$ National Center for Spaceweather Monitor and Forecast, Chinese Meteorological \\ Administration, Beijing 100081
}

\begin{abstract}
Based on the solar energetic particle (SEP) data from ACE and GOES satellites, the acceleration of SEP by CME-driven shock in interplanetary space was investigated. The results showed that the acceleration process of SEP by the Bastille CME-driven shock ran through the whole space from the sun to the magnetosphere. The highest energy of SEP accelerated by the shock was greater than $100 \mathrm{MeV}$. A magnetic bottle associated with the CME captured a lot of high energy particles with some of them having energy greater than $100 \mathrm{MeV}$. Based on magnetic field data of solar wind observed by ACE data, we found that the the magnetic bottle associated with the Bastille CME was the sheath caused by the CME in fact.
\end{abstract}

Keywords. shock waves, Sun: coronal mass ejections (CMEs), particle emission

\section{Introduction}

Reames (1999) introduced SEP events' properties in great detail. He think that there are two kinds of SEP events, impulsive type related to flare and gradual type related to fast shock driven by a CME. An important point of view of Reames(1999,2002) is that large SEP events are only related to CME-driven shock. Gopalswamy's (2002) view of point is that the interaction between two CMEs' came from the same region within $24 \mathrm{~h}$ play an important role for large SEP events. All these work mainly emphasize CME's role and dismissed flare's function. Recently Richardson (2003) argued that the CME interaction may be not important for accelerating major solar energetic events. Some results (Cohen et al., 1999; Mazur et al., 1999) showed that flare may provide some seed particles for CME-driven shock. Torsti et al. (2001) pointed out that the SEP event on 1999 May 9 was a hybrid event, the flare first accelerated particles and then CME accelerated the particles produced by the flare. Mewaldt et al. (2003) pointed out that remnant of interplanetary material from earlier impulsive can't provide sufficient Fe in hybrid events, which also implied that flare may provide seed particles for CME-driven shock. Kallenrode (2003) suggested SEP events should consist of flare accelerate particles (FAPs) and CME-driven shock accelerate particles (CSAPs), with the only FAPs or only CSAPs being the limiting case.

The solar active region AR9077 produced a X5.7 flare and a intensive coronal mass ejection(CME) causing very strong disturbance in Sun-earth connection space on 2000 July 14. This day was called Bastille day and the solar event on that day was called Bastille Day Event, CME on 2000 July 14 was called Bastille CME in this paper. The event has been paid attention greatly in the world. Many paper have been devoted to

$\dagger$ Present address: 20A Datun Road, Chaoyang District, Beijing, China 
the event study. Reames et al. (2001) analyzed the heavy ion abundance and spectra of the event. Bieber et al. (2002) investigated the intensity-time and anisotropy-time profiles and pitch-angle distributions of energetic protons near Earth; Tylka et al. (2001) explained the energy spectra of Fe by shock acceleration from solar wind suprathermals and small ( $\sim 5 \%)$ admixture of remnants flare particles. Maia et al (2001) study the energetic electron on Bastille Day Event by analyzing the radio data observed by Nancay radio heliograph. Le et al (2004) ever studied the moving direction of the Bastille CME by using galactic cosmic ray data. M $\ddot{A}$ KEL $\ddot{A}$ et al. (2001) analyzed the time variation of $2.7-3.3 \mathrm{MeV} \mathrm{nucl}{ }^{-1}$ and $10-13 \mathrm{MeV} \mathrm{nucl}^{-1}$ protons and some heavy ions at energy 8.5 $15 \mathrm{MeV}$. They thought that the magnetic tubes were filled with flare-related particles. Tang et al. (2003) suggested that the Bastille Day event was a hybrid event. Very recently Tang (2004 Sep.) found that the protons were firstly accelerated by electric field in the magnetic reconnection region by analyzing the data of gama ray and EIT/SOHO. So it seems that the event is still not completely understood.

Here we report that the data of high energy protons with energy greater than $10 \mathrm{MeV}$ and $30 \mathrm{MeV}$ in interplanetary space observed by Advanced Composition Explorer (ACE). We also analyze the energetic particles with energy greater than $10 \mathrm{MeV}, 30 \mathrm{MeV}, 50 \mathrm{MeV}$ and $100 \mathrm{Mev}$ observed by Goes satellite. We study the CME-driven shock's role in acceleration of Bastille Day SEP Event. Finally we investigate which part associated with the Bastille CME captured a lot of higher energy particles with some of them having energy greater than $100 \mathrm{MeV}$.

\section{Data Analysis}

Because only CME or flare may produce or accelerate solar energetic particle, so we firstly present the flare and CME's information related to Bastille Day SEP event. On 2000 July 14, a big flare flare X5.7 began at 10:03UT, peaked at 10:24UT and ended at 10:43, shown in Fig.1. There were no other important flare except the X5.7 during 14-16, July 2000. Based on the flare classification given by Pallavicini et al. (1977), the flare X5.7 was a gradual flare. While the whole duration of the flare is shorter than 1 hour, so the flare belonged to impulsive flare based on the definition of a flare by its time structure.

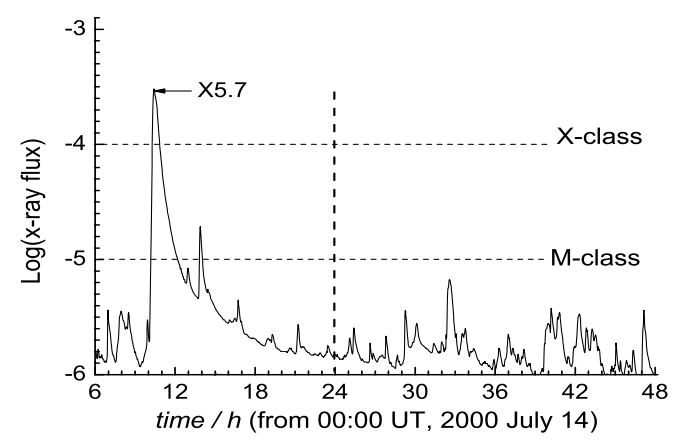

Figure 1. solar flare during 14-16, Jul 2000

The CMEs' came from the AR9077 on 2000 July 13 and Bastille day are listed below (copy from Gopalswamy's (2002) paper ). CME1's onset was 14 hour and $24 \mathrm{~m}$ earlier than CME2's onset.

The data from the SIS instrument on ACE during 14 15, July 2000 are shown in Fig.2. From Fig.2 we can see that the proton flux with energy greater than $10 \mathrm{MeV}$ 
Table 1. 2000 Jul. 13 and 14's CME information

\begin{tabular}{|c|c|c|c|c|}
\hline Date & CME | & Onset time & Width & |Speed $(\mathrm{km} / \mathrm{s})$ \\
\hline | 2000 Jul & CME1| & 20:30UT & 62 & 839 \\
\hline 2000 & E2 & 10:54UT & 360 & 1674 \\
\hline
\end{tabular}
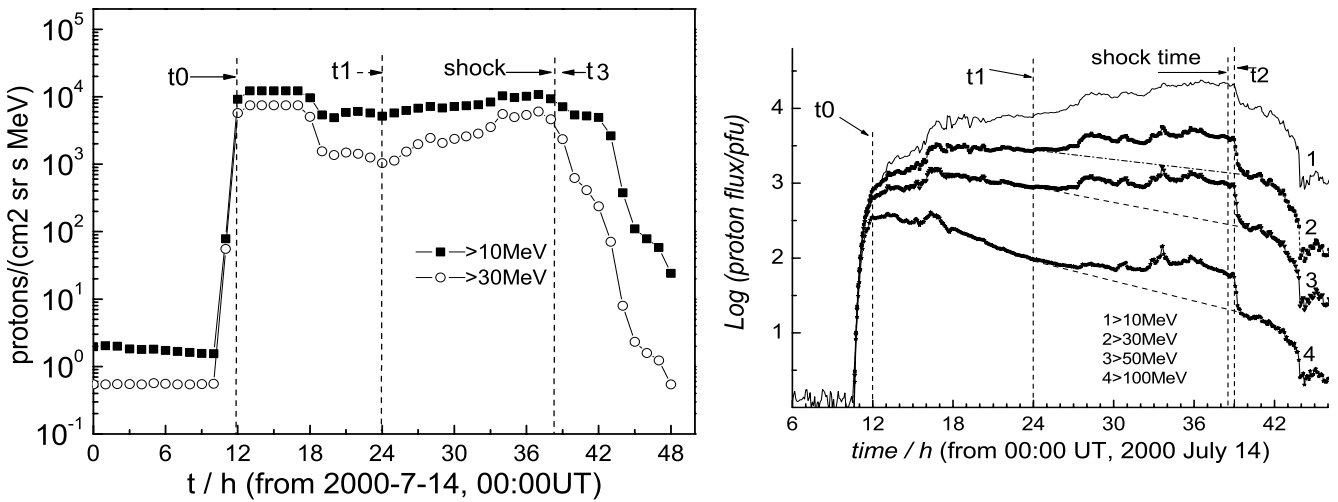

Figure 2. the SEP data observed by ACE Figure 3. the SEP data observed by GOES 8

and $30 \mathrm{MeV}$ reached saturation no long after the onset of the SEP event. At about 2000 July 14 18:00UT, the proton flux with energy greater than $10 \mathrm{MeV}$ and $30 \mathrm{MeV}$ decreased which means that the shock driven by the CME2 accelerated particles very intense during 11:00UT 18:00UT, July 14, 2000. With the deceleration of the CME2 as it traveling outward, the ability of CME2-driven shock acceleration of SEP decreased. So the enhancement of SEP flux from t1 to shock time must not be caused by shock driven by the Bastille CME. Because there was no important flare around the 24:00UT and also no CME around that time, so the enhancement must be caused by the CME itself. The SEP flux observed by GOES satellite increased also from t1 for four energy channels including the channel of greater than $100 \mathrm{MeV}$ shown in Fig.3. After shock passed ACE, the SEP flux was still high during the period from shock time to $\mathrm{t} 3$ shown in Fig. 2, so there were large number of energetic particles captured by the magnetic bottle. Based on the ACE data, the location of the magnetic bottle relative to the CME-driven shock and CME itself was shown in Fig.4.

\section{Summary and Discussion}

The Bastille Day CME-driven shock had been accelerating SEP with energy greater than $100 \mathrm{MeV}$ during the period from the onset time to the moment that shock reached the the magnetosphere. The magnetic bottle associated the Bastille CME captured a lot of high energy particles with some of them having energy greater than $100 \mathrm{MeV}$. The location of the magnetic bottle associated with the Bastille CME was behind the shock but in front of the magnetic cloud.

In fact the magnetic bottle associated with Bastille CME was the sheath caused by the CME. The Bastille CME-driven shock accelerated SEP very intensively causing the SIS SEP instrument saturation during the period from the onset time of SEP to about 18:00UT, 2000 July 14. After that the SEP flux observed by ACE decreased for a while shown in Fig.2. The SEP flux with energy greater than $10 \mathrm{MeV}, 30 \mathrm{MeV}$ and $50 \mathrm{MeV}$ observed by GOES satellite didn't decline obviously around 18:00UT, 2000 July 14. This 


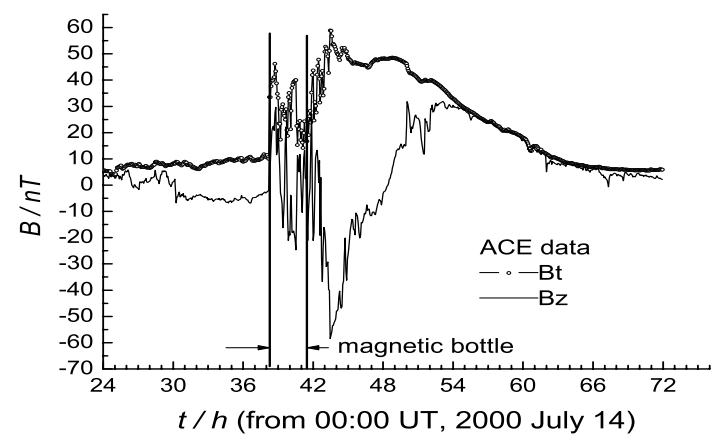

Figure 4. The location of magnetic bottle associate the Bastille CME

means that the charged particles with energy greater than $10 \mathrm{MeV}, 30 \mathrm{MeV}$ and $50 \mathrm{MeV}$ can stay in geosynchronous orbit for some time. There was big difference between the SEP data in geosynchronous orbit observed by GOES satellite and the SEP data in interplanetary space observed by ACE satellite for the particles with energy greater than $10 \mathrm{MeV}$ and $30 \mathrm{MeV}$.

\section{Acknowledgements}

We thank Dr. Allan J. Tylka and Professor Ruffolo David for their helpful suggestions and we have made some revision for the paper. The data used in this paper are downloaded from http://cdaweb.gsfc.nasa.gov/cdaweb/sp_phys/ and http://spidr.ngdc.noaa.gov/. This work is supported by National Nature Science Foundation of China (through Grant No. 10073013) and National Center for Spaceweather Monitor and Forecast, Chinese Meteorological Administration.

\section{References}

Bieber, J.W., Droge, W., Evenson, P.A., et al., 2002, ApJ 567, 622B

Cohen,C.M.S., Cummings, A.C., Leske, R.A., 1999, , Geophys. Res.Lett. 26, 149

Duldig, M.L., Bombardieri, D.J., Humble, J.E., 2003, 28th ICRC 6, 3389

Gopalswamy, N., Yashiro, S., Michalek, G., 2002, ApJ 572, L103

Kallenrode, M.B., 2003, J Phys. Nucl. Part. Phys. 29, 965

Kocharov, L., Torsti, J., 2002, Solar Physics 207, 149

Le, G.M., Ye, Z.H., 2004, Chin. J. Space Sci. 24, 15 (in Chinese)

Maia, D., Pick, M., Hawkins, III S.E., et al., Solar Phys. 204, 199

Makela Pertti, Torsti Jarmo., Solar Phys. 204, 215

Mewaldt, R.A., Cohen, C.M.S., Mason, G.M., et al., 2003, 28th ICRC 6, 3229

Mazure, J.E., Mason, G.M., Looper, M.D., et al., 1999, Geophys. Res.Lett. 26, 173

Pallavicini, R., Serio, S., Vaiana, G.S., 1977, ApJ 216, 108

Raeder, J., Wang, Y.L., 2001, Solar Phys. 204, 325

Reames, D.V., 2001, Astrophys. J. 548, L233

Reames, D.V., 1999, Space Science Review 90, 413

Reames, D.V., 2002, ApJ 571, L63

Richardardson, I.G., Lawrence, G.R., Haggerty, D.K., et al., 2003, GRL. 30(12), 8014

Shinichi, W., Manabu, K, Takashi, W., 2001, Solar Phys. 204, 425

Tang, Y.H., Dai, Y., 2003, Adv. Space Res., 12, 2609

Tang, Y.H., private communication, 2004 Sep.

Torsti, J., Kocharov, L., Innes, D.E., et al., 2001, A\&A A 365, 198

Tylka, A.J., Cohen, C.M.S., Dietrich, W.F., 2001, ApJ 558, L59 